\title{
Flock House Virus RNA1 with a Long Heterologous Sequence at the 3 '-end Can Replicate in Mammalian Cells and Mediate Reporter Gene Expression
}

\author{
Doyeong Kim and Tae-Ju Cho* \\ School of Life Sciences, Chungbuk National University, Cheongju 28644, Republic of Korea
}

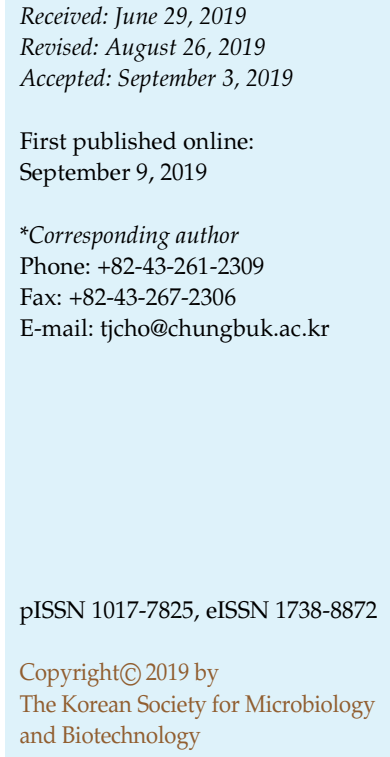

\section{Introduction}

A previous study showed that foreign RNAs can be packaged into turnip yellow mosaic virus (TYMV) when they are expressed as a subgenomic RNA (sgRNA) under the influence of an additional sgRNA promoter, tymobox [1]. TYMV is an icosahedral plant virus belonging to the genus Tymovirus. The TYMV virion contains a single $6.3 \mathrm{~kb}$ positive-sense RNA as a monopartite genome [2]. A $0.8 \mathrm{~kb}$ sgRNA encoding coat protein $(\mathrm{CP})$ is also packaged in the virion. Using this system, we were able to package Flock House virus (FHV) RNA1 in TYMV. The encapsidated FHV RNA had the TYMV CP sequence at the $3^{\prime}$-end and four additional nucleotides at the $5^{\prime}$-end [3]. FHV is a positivestrand RNA virus belonging to the Nodaviridae family and it also has the smallest genome among animal viruses. The and it also genome consists of two RNAs, the larger one of which, $3.1 \mathrm{~kb}$ RNA1, encodes a viral replicase that can replicate independently of RNA2 [4]. RNA2 contains a gene for a viral coat protein precursor. A small FHV sgRNA, RNA3, with two overlapping reading frames encoding B1 and B2 proteins, is generated from RNA1 during replication [5]. B2 is known to be a potent RNAi suppressor [6, 7].

Recently, plant viruses have been investigated as a potential gene delivery vector. Plant viruses are attractive since they do not infect animal cells and can be produced in large amounts at low cost. Tobacco mosaic virus (TMV) was the first case of such a study. Smith et al. [8] incorporated a TMV origin of assembly (Oa) sequence into Semliki Forest virus (SFV) RNA, and successfully encapsidated the recombinant SFV RNA into TMV particles using an in vitro assembly system. The pseudovirus particles expressed reporter genes when introduced into baby hamster kidney (BHK) cells. In another study, Zhou et al. [9] demonstrated that Oa-adapted FHV RNA1 can be encapsidated by TMV CPs in planta. The transgene marker successfully elicited immune response when mice were injected with the FHV RNA1. Similarly, cowpea chlorotic 
mottle virus (CCMV) pseudovirus particles containing Sindbis virus RNA were prepared through in vitro assembly of CCMV CPs in the presence of the animal virus RNA [10]. The recombinant Sindbis virus RNA was shown to replicate and express reporter genes in BHK21 cells.

We assessed the potential of utilizing the natural packaging mechanism of TYMV to package therapeutic RNA molecules. As a proof of concept, we attempted to package a recombinant FHV RNA1 containing the eGFP gene as a reporter in TYMV. As mentioned earlier, the recombinant FHV RNA1 was encapsidated into TYMV [3]. The encapsidated FHV RNA1 is, however, different from the authentic FHV RNA, in that it has terminal extensions both at the $5^{\prime}$ - and $3^{\prime}$-ends. The four-nucleotide extension at the $5^{\prime}$-end was inevitable, since the four-nucleotide sequence is shared by both the sgRNA promoter tymobox [11] and the overlapping replicase gene. The recombinant FHV RNA1 has a long extension corresponding to the $800 \mathrm{~N}$ TYMV CP sequence at the $3^{\prime}$-end. Although FHV RNA without the TYMV sequence was generated during infection of TY-FHV(eGFP) in plant cells, only the FHV RNA having the TYMV CP sequence at the $3^{\prime}$-end was preferentially encapsidated into TYMV [3].

We were interested in whether the recombinant FHV RNA1 with extensions at both ends could replicate efficiently in host cells, since it was reported that FHV RNA1 replication was very sensitive to the extension at the termini, particularly at the 5 -end [5]. Two and 10 nucleotide additions at the $5^{\prime}$-end decreased replication by $80 \%$ and $98 \%$, respectively. Although 3 -terminal extension was less crucial than $5^{\prime}$-terminal extension, 12 nucleotide additions at the $3^{\prime}$-end resulted in a more than $40 \%$ decrease in replication. We first examined in Nicotiana benthamiana whether the recombinant FHV RNA1 is capable of replication. The result showed that the recombinant FHV RNA can replicate and express eGFP [12]. We extended the study to mammalian cells and showed that the recombinant FHV RNA1 can also replicate in BHK cells. Interestingly, we also observed eGFP abundance in BHK21 cells in a temperature-dependent manner. Overall, our findings highlighted that TYMV can be used to package recombinant FHV RNAs that can replicate and express foreign genes upon delivery to mammalian host cells.

\section{Materials and Methods}

\section{DNA Constructs}

The TY-FHV(eGFP) construct was described by Kim et al. [3]. The pCB FHV-CP, pCB FHV(eGFP), and pCB FHV(eGFP)-CP constructs, which were used to introduce TYMV replicons to $N$. benthamiana, were described by Kim et al. [3]. The DNA templates used for in vitro transcription were prepared using these constructs. To prepare DNA templates for FHV RNA and FHV(eGFP) RNA, PCR was performed using the following forward primer containing the T7 promoter sequence: $5^{\prime}$ TAATACGACTCACTATAGGGAATAGTTTGAAACAAATAAAA $C A G A A A-3^{\prime}$ (the T7 promoter sequence is indicated in boldface, and the FHV sequence is italicized). The forward primer used to prepare $\mathrm{wt}^{*} \mathrm{FHV}$ RNA was as follows: 5'-TAATACGACTCA CTATAGGTTTGAAACAAATAAAACAGAAA-3'. The reverse primer used was 5'-ACCTCTGCCCTTTCGGG-3'. To yield DNA templates for FHV-CP and FHV(eGFP)-CP RNA synthesis, the following was used as a reverse primer: $5^{\prime}$-GGTTCCGATGAC CCTCGGAAG A-3'.

\section{In Vitro Transcription}

For in vitro transcription, the mMESSAGE mMACHINE Kit (Ambion, Korea) was used. DNA templates were used to prepare in vitro transcribed RNA and PCR was performed as described above. The PCR products were then subjected to in vitro transcription, followed by attachment of 5 -cap structures. The capped RNA transcripts were precipitated with $\mathrm{LiCl}$, quantified by UV spectrophotometry, and stored at $-20^{\circ} \mathrm{C}$.

\section{Transfection of BHK Cells}

BHK21 cells were maintained in RPMI 1640 media (Thermo Fisher, USA) containing 10\% fetal bovine serum (FBS; ATCC, USA). BHK21 cells grown to confluency were transferred to a 6well plate at a density of $2 \times 10^{5}$ cells per well, and incubated for $16 \mathrm{~h}$. After washing the cells twice with phosphate-buffered saline (PBS), $500 \mu \mathrm{l}$ of Opti-MEM media containing $2 \mu \mathrm{g}$ of RNA transcripts and $6 \mu \mathrm{l}$ of Lipofectamine 2000 (Thermo Fisher) was added to the cells. After incubating the cells at $37^{\circ} \mathrm{C}$ and $5 \% \mathrm{CO}_{2}$ for $4 \mathrm{~h}$, the transfection media were replaced with fresh RPMI 1640 media. The plate was then moved to an incubator and incubated for $48 \mathrm{~h}$ at $28^{\circ} \mathrm{C}$ or $30^{\circ} \mathrm{C}$. Fluorescence images of BHK21 cells were obtained via a Nikon E-200 microscope (Nikon, Japan) or Leica DE/DM18 (Leica, Germany). Cell nuclei were stained with Hoechst 33258 (Sigma-Aldrich, USA).

Cell viability was analyzed $48 \mathrm{~h}$ after transfection by MTT [3(4,5-dimethylthiazol-2-yl)-2,5-diphenyltetrazolium bromide] assay as described by Kim et al. [13]. The experiments were performed in triplicate.

\section{Analysis of RNA}

Total RNA was isolated from frozen $N$. benthamiana leaf samples using Easy-Red (Intron, Korea). Total RNA from BHK21 cells was isolated using Trizol (Invitrogen, USA), followed by isopropanol precipitation. The ribonuclease protection assay for encapsidated RNA in TYMV virions and northern blot analysis were performed as previously described [14]. RNA samples were size-fractionated by electrophoresis on a $1 \%$ agarose gel, and were transferred onto 
Hybond N+ membranes (GE Healthcare, UK). The blots were hybridized with a DIG-labeled DNA probe representing the TYMV CP ORF, the FHV sgRNA, or both.

\section{RNA Ligase-Mediated Rapid Amplification of cDNA Ends (RLM-RACE)}

RLM-RACE was performed using the FirstChoice RLM-RACE Kit (Thermo Fisher). Total RNA samples were treated with calf intestine alkaline phosphatase (CIP) to remove the $5^{\prime}$-phosphates from all RNA molecules with free $5^{\prime}$-phosphates. RNA samples were then treated with tobacco acid pyrophosphatase (TAP) to remove the $5^{\prime}$ cap structures of RNAs, leaving 5'-monophosphates on mRNAs and viral RNAs. For 5' RLM-RACE, oligoribonucleotides were ligated to the $5^{\prime}$-ends of the decapped RNAs. After reversetranscription, the $5^{\prime}$-ends of FHV RNAs were PCR-amplified using the $5^{\prime}$ RACE outer primer (5'-GCTGATGGCGATGAATGAACACTG$\left.3^{\prime}\right)$ containing the adaptor sequence as a forward primer and FHV307R (5'-TGAAACGGCGTGCCCGTTGTC-3') or FHV3016R (5'-CACTTCCGGTTGTTGGAAGG-3') as a reverse primer. For 5'-3' RLM-RACE, the enzyme-treated RNA samples were ligated to each other without RNA adaptors and were used for cDNA synthesis. The cDNAs were then used to amplify the 5'- and 3'ends of FHV RNA1 using the following primers: FHV3061F ( $5^{\prime}$ GGCTCTTAGGAGCACCCACA-3') and FHV307R. The PCR products were cloned into a TA-cloning vector, and the resulting clones were selected at random for sequence analysis.

\section{Western Analysis}

BHK21 cells were resuspended in the SDS-PAGE loading buffer after being washed twice with PBS, and boiled for $3 \mathrm{~min}$. Proteins were separated by $12.5 \%$ SDS-PAGE followed by western blot analysis. eGFP was detected using rabbit anti-GFP antibody (Santa Cruz Biotechnology, USA) in conjunction with HRP-conjugated goat anti-rabbit IgG (Bio-Rad, USA). The blot was developed by a chemiluminescent detection method, using Luminata Forte (Millipore, USA). Glyceraldehyde-3-phosphate dehydrogenase (GAPDH) was detected using mouse anti-GAPDH antibody (Sigma, USA).

\section{Results}

\section{Replication of Recombinant FHV RNA in Mammalian Cells}

The construct that was used to produce a recombinant FHV RNA1 containing an eGFP gene as a TYMV sgRNA in $N$. benthamiana is shown in Fig. 1A. The larger TYMV sgRNA, sgRNA1, contained the FHV sequence and was encapsidated into TYMV virions along with the smaller sgRNA2 [3]. To determine if recombinant FHV RNAs containing the heterologous sequence can replicate in mammalian cells, RNAs were prepared by in vitro transcription using the DNA templates shown in Fig. 1B.
A

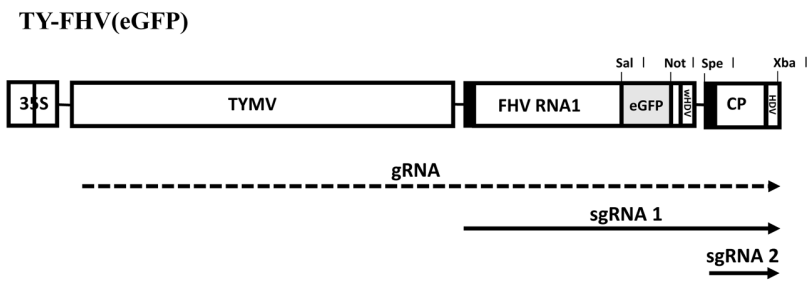

B

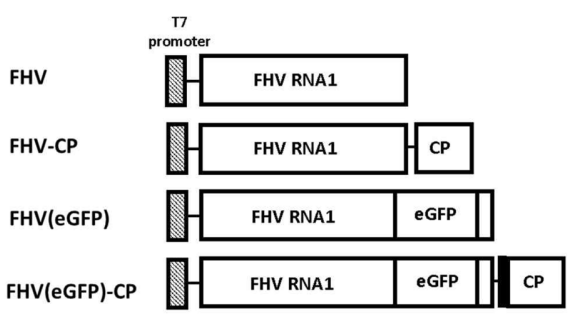

C $\quad$ FHV

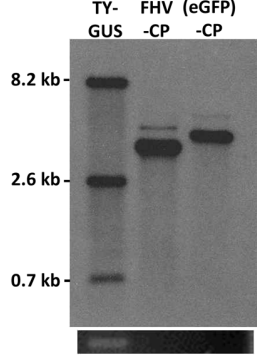

Fig. 1. DNA constructs used in this study.

(A) The TY-FHV(eGFP) construct used to package FHV RNA into TYMV virions. Preparation of TY-FHV(eGFP) was described previously (Kim et al., 2014). Briefly, the FHV RNA1 sequence was placed downstream of the tymobox (black box), a sgRNA promoter. The eGFP sequence was inserted into the B2 ORF of the FHV genome. The TYMV genome was placed downstream of double $35 \mathrm{~S}$ promoters of Cauliflower mosaic virus (CaMV). Genomic RNA (gRNA) is depicted in broken line to indicate that the amount of encapsidated RNA is negligible compared to subgenomic RNAs (sgRNAs). (B) DNA templates used for in vitro transcription. Templates were prepared using PCR amplification, in which the forward primers included the T7 promoter sequence. (C) Northern blot analysis of in vitro transcribed FHV RNAs. In vitro transcribed RNAs $(1 \mu \mathrm{g})$ from FHV-CP and FHV(eGFP)-CP templates were size-fractionated on a $1 \%$ agarose gel and examined by northern blot analysis, using the DIG-labeled probe targeting TYMV CP sequence. Total RNA isolated from the leaf agroinfiltrated with TY-GUS, which expresses a $1.8 \mathrm{~kb}$ GUS gene as a sgRNA, was included as a size marker. The panel below the northern blot shows $25 \mathrm{~S}$ rRNA stained with EtBr.

The template was prepared by PCR, and the T7 promoter was added to the FHV sequence. Since it was anticipated that four nucleotides would be added to the 5 '-end of the FHV RNA packaged in TYMV virions, AATA was added to the $5^{\prime}$-end. GGG was also included at the 5 '-end to enhance the transcription of template DNA by T7 RNA polymerase. Fig. 1C shows the in vitro transcribed products derived from two templates that contained the TYMV CP sequence at the $3^{\prime}$-end.

In vitro transcribed RNAs were transfected into BHK21 cells, and 2 days after transfection, RNA samples were analyzed. In this experiment, RNA with one G at the 5 '-end 
( $\left.w t^{*} \mathrm{FHV}\right)$ was also included to compare the replication of recombinant FHV RNA with that of wild-type FHV RNA1, based on the previous observation that FHV RNA1 with one $\mathrm{G}$ added to the $5^{\prime}$-end was comparable to wild-type FHV RNA1 [5]. We demonstrated that recombinant FHV RNA with the TYMV CP sequence generates replicating
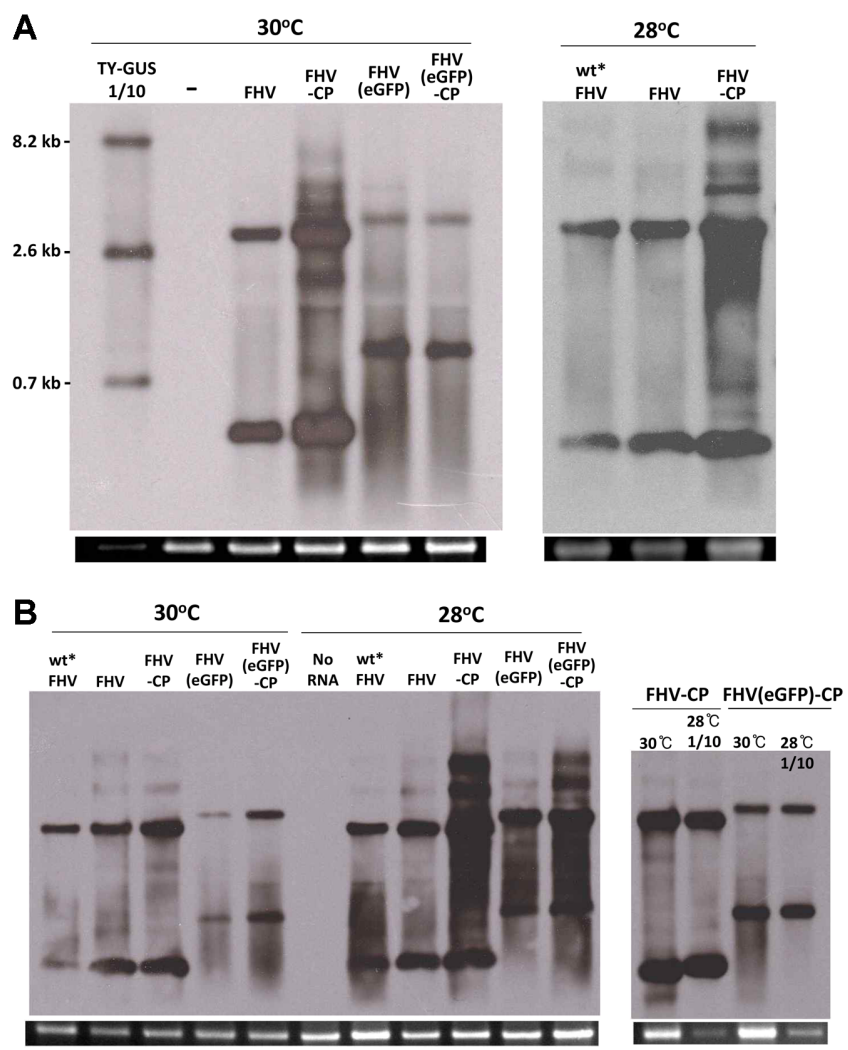

Fig. 2. Replication of FHV RNA in BHK21 cells.

(A) Northern blot analysis of FHV RNA replication. FHV constructs were subjected to in vitro transcription and the RNA products were transfected into BHK21 cells with the aid of Lipofectamine 2000. Total RNA was isolated at $48 \mathrm{~h}$ post-transfection and examined by northern blot analysis as described in Fig. 1C, except that the probe used was a mixture of DIG-labeled DNAs targeting FHV sgRNA and TYMV CP sequences. Experiments were performed in triplicate, and a representative blot is shown. The right panel shows the replication of FHV RNA1, where one G nucleotide was included at the 5 '-end of FHV RNA1 ( $\left.w t^{*} \mathrm{FHV}\right)$. The $w t^{*} \mathrm{FHV}$ RNA was reported to replicate as efficiently as wild-type FHV RNA1 [5]. (B) Replication at two different temperatures. Transfected BHK21 cells were incubated at two different temperatures, $28^{\circ} \mathrm{C}$ and $30^{\circ} \mathrm{C}$, and the results were compared. Total RNA extracted from the BHK21 cells incubated at either temperature was examined by northern blot analysis using the DIG-labeled DNA targeting FHV sgRNA. The right panel shows the result of northern blot hybridization using the " $28^{\circ} \mathrm{C}^{\text {" RNA samples }}$ diluted 1:10 for comparison.
RNAs (Fig. 2A). The presence of FHV sgRNA clearly indicated that the introduced RNA replicated. Thus, the heterologous sequence at the 3 '-end of FHV RNA does not inhibit replication.

The recombinant FHV-CP RNA gave rise to a similar or greater level of RNA when compared to FHV RNA without the TYMV CP sequence. In the case of FHV RNA containing the eGFP insert, the recombinant FHV RNA exhibited less replication compared with the FHV RNA without the eGFP gene, indicating that presence of a foreign sequence within the FHV RNA inhibited viral RNA replication to some extent. It was also observed that the replication of FHV RNA1 with an additional GGGAATA at the $5^{\prime}$-end and no $3^{\prime}$ TYMV CP sequence was comparable to that of wild-type (Fig. 2A, right panel), showing that seven-nucleotide extension at the $5^{\prime}$-end did not affect the FHV RNA1 replication.

\section{eGFP Expression}

We then examined eGFP expression in the transfected BHK21 cells by fluorescence microscopy. Unexpectedly, only a few cells expressed eGFP with less than $0.1 \%$ of the transfected cells emitting fluorescence. Initially, we presumed that the result could be ascribed to a defective B2 protein. A previous study showed that B2 plays a vital role in viral replication [15]; FHV mutants lacking the ability to produce B2 yielded negligible amounts of progeny virus. An interesting observation was that the cells infected with the B2 mutant contained very small amounts of coat protein despite an abundance of FHV RNA. This study showed that B2 protected FHV RNA2 by preventing RNA recruitment into a cellular structure where it was translationally silenced.

Since a similar pattern (abundant RNA and little protein) was observed in our study, we hypothesized that B2 protein became defective, possibly due to eGFP insertion. In our constructs, the eGFP gene was inserted near the end of the B2 ORF, resulting in the production of B2 proteins lacking six amino acids at the $\mathrm{C}$-terminal end. We initially assumed that the deletion may have affected B2 function. If the $\mathrm{B} 2$ protein was defective due to the insertion of eGFP, it would explain the discrepancy between RNA and protein levels; however, Petrillo et al. [15] also reported that the removal of 32 amino acids at the C-terminal end of B2 did not harm the function of the protein. Our result was also inconsistent with the findings of Maharaj et al. [16], who used the same site of the FHV genome for eGFP insertion and observed strong fluorescence in cells transfected with the recombinant FHV RNA. 
We then noticed that the incubation temperature was set at $30^{\circ} \mathrm{C}$. To confirm whether incubation temperature affected eGFP synthesis, we changed the temperature to $28^{\circ} \mathrm{C}$. FHV RNA replication and eGFP expression at $28^{\circ} \mathrm{C}$ was compared with our findings at $30^{\circ} \mathrm{C}$ (Figs. $2 \mathrm{~B}$ and 3). At $28^{\circ} \mathrm{C}$, FHV RNA replication was higher compared with replication at $30^{\circ} \mathrm{C}$ (Fig. 2B). Consistent with this result, at $28^{\circ} \mathrm{C}$, more BHK21 cells expressed eGFP (Fig. 3A). Western blot analysis of eGFP also confirmed this result (Fig. 3B). However, the difference in protein levels at two temperatures seemed to be far greater than expected from the RNA levels.
We posited that this interesting observation might be related to B2 protein since a previous study suggested that B2 protein could protect its own mRNA, FHV RNA3 [15]. Thus, since the eGFP was expressed as a fusion protein with B2, an explanation could be that decreased eGFP expression at $30^{\circ} \mathrm{C}$ might be an outcome of the interaction between $\mathrm{B} 2$ and eGFP. At $30^{\circ} \mathrm{C}$, the conformation of B2eGFP may have been altered in such a way that eGFP in the B2-eGFP fusion protein interfered with the activity of B2. Alternatively, the temperature effect might simply reflect the natural expression pattern of B2 at different temperatures.
A

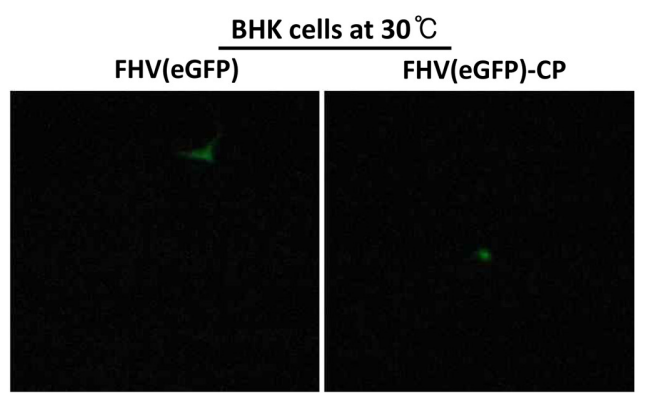

BHK cells at $28^{\circ} \mathrm{C}$

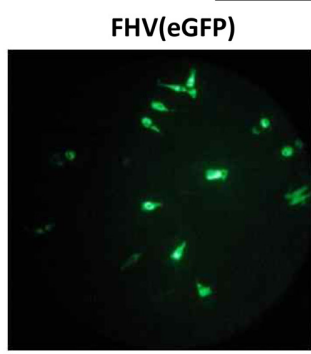

FHV(eGFP)-CP
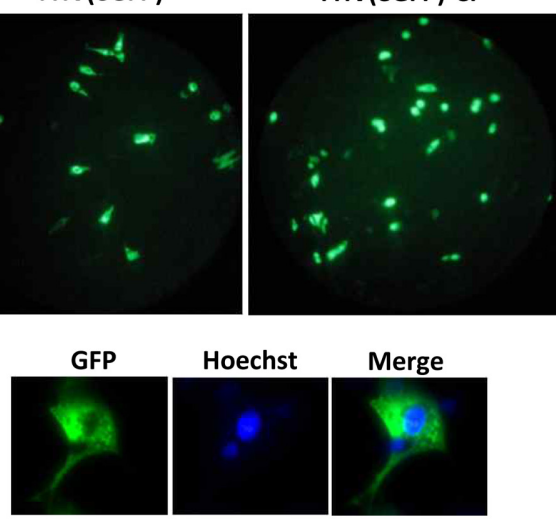

B

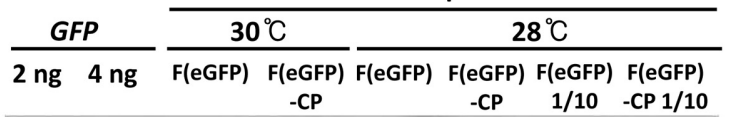

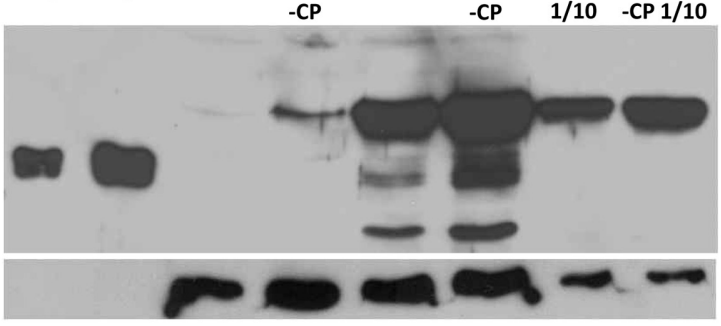

$30^{\circ} \mathrm{C}$

$28^{\circ} \mathrm{C}$

F(eGFP) F(eGFP) F(eGFP) F(eGFP) F(eGFP) F(eGFP)

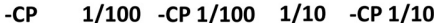
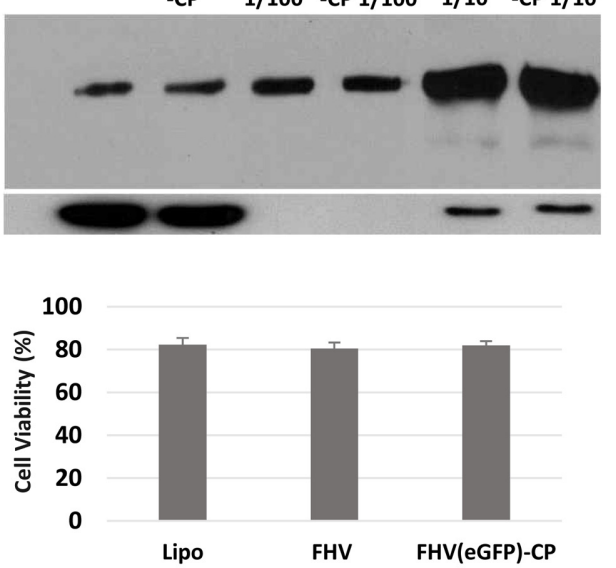

Fig. 3. eGFP expression in BHK cells.

(A) Fluorescence microscopy of eGFP-expressing cells. Transfected BHK21 cells were incubated at two different temperatures, $28^{\circ} \mathrm{C}$ and $30^{\circ} \mathrm{C}$, and the results were compared. Top and middle panels show transfected cells incubated at $30^{\circ} \mathrm{C}$ and $28^{\circ} \mathrm{C}$, respectively. Note that the images from the BHK21 cells incubated at $30^{\circ} \mathrm{C}$ were the result of careful searching for fluorescence-emitting cells. Thus, the number of fluorescent cells in the " $30^{\circ} \mathrm{C}$ " sample should be less than that anticipated from the images. Bottom panel shows representative images of the cells whose nuclei were stained with Hoechst 33258 (blue). (B) Western blot analysis of the eGFP protein. The transfected cells were washed and then boiled for 5 min after 2X SDS-PAGE loading buffer was added. eGFP was detected using an anti-eGFP antibody and anti-rabbit HRP conjugate. 1:10 and 1:100 diluted " $28^{\circ} \mathrm{C}$ " samples were included for comparison. Experiments were repeated at least three times, and representative results are shown. FHV(eGFP) is abbreviated as F(eGFP). The blot showing GAPDH was included in the bottom panel to show a loading control. (C) Cell viability examined by MTT assay. BHK21 cells transfected with FHV RNA1 (FHV) or FHV(eGFP)-CP RNA were examined by MTT assay after incubation at $28^{\circ} \mathrm{C}$ for 48 $\mathrm{h}$. The sample treated with Lipofectamine only (Lipo) was included as a control. MTT value of untreated cells after $48 \mathrm{~h}$ of incubation was taken as $100 \%$. The measurements were performed in triplicate, and the results are represented as mean \pm SD (error bar). 
MTT assay was performed to examine the viability of the cells $48 \mathrm{~h}$ after transfection. The result showed that the MTT absorbance values of the cells treated with Lipofectamine only were not significantly different from those of the cells transfected with FHV RNA1 $(n=5 ; p>0.5)$ or FHV(eGFP)CP RNA $(n=5 ; p>0.8)$ (Fig. 3 C), indicating that FHV RNA replication and eGFP expression in BHK cells resulted in negligible effect on cell viability.

\section{Sequence Analysis of Replication Products}

We examined whether the replication products from the FHV(eGFP)-CP construct were identical to authentic FHV RNA, since the template has additional nucleotides at the $5^{\prime}$ - and $3^{\prime}$-ends. Because the $3^{\prime}$-end of the RNA of a positive-strand RNA virus is the start site of (-)-RNA synthesis, the 3 '-terminal sequence is crucial for viral RNA replication. Previously, Albarino et al. [17] reported that 108-nucleotide sequence at the 3'-end of FHV RNA1 was sufficient to ensure replication. Eckerle et al. [18] also examined the production of FHV sgRNA, RNA3, and reported that a 58-nucleotide sequence at the 3 -end was required for the replication of RNA3. Since FHV RNA3 is also a template for FHV replicase and the $3^{\prime}$-end of RNA3 is the same as that of RNA1, the 58-nucleotide 3'-terminal sequence seems to be important for the replication of both FHV RNA1 and RNA3; however, there have been conflicting observations on the importance of this $3^{\prime}$-terminal sequence. Ball [5] reported that deletion of five nucleotides at the $3^{\prime}$-end abolished RNA1 production in BHK21 cells. In contrast, a recent in vitro study by Wu et al. [19] showed that FHV RNA1 synthesis initiation could tolerate the loss of two nucleotides at the $3^{\prime}$-end. They also reported that deletion of up to 29 nucleotides at the $3^{\prime}$-end did not prevent RNA1 synthesis. This indicates that the 3'-terminal sequence may not be crucially important for replication.

If the 3'-terminal FHV sequence is not crucial for replication, it could be that the FHV replicase initiates RNA synthesis at random. This conjecture could be tested by sequence analysis of replication products; however, sequence analysis of FHV RNA replication products has not previously been reported. In this study, we could examine how FHV replicase responds when there is an extension at both ends of the template RNA. To address these issues, we examined the sequences of the replication products generated in BHK cells from FHV(eGFP)-CP by performing RLM-RACE (Fig. 4A). Since the $5^{\prime}$-end of viral RNA turns into the $3^{\prime}$-end on (-)-strand RNA, the 5'terminal sequence of viral RNA was also examined. The $5^{\prime}$ cap structures of viral RNAs were removed by treatment with CIP and TAP, leaving $5^{\prime}$-monophosphates on the viral RNA. The 5'-ends of the FHV RNA were PCR-amplified after ligation of oligoribonucleotides to the $5^{\prime}$-ends of the decapped RNAs, followed by reverse-transcription. For the examination of the $3^{\prime}$-ends of FHV RNA, the enzymetreated RNA samples were self-ligated using T4 RNA ligase without added RNA oligomers. The regions comprising the 5'- and 3'-ends of FHV RNA were then PCR-amplified after cDNA synthesis.

After cloning the PCR-amplified DNA products into plasmid vectors, clones were randomly selected, and their sequences were analyzed. The results are shown in Fig. 4B. In the case of FHV RNA1, six out of the 19 clones of 5'-end sequences had small deletions. Thirteeen clones had the same RNA1 5'-ends. None of the $5^{\prime}$-ends of the clones analyzed had all or part of the four nucleotides (5'-AATA$3^{\prime}$ ) added to the FHV sequence. The $3^{\prime}$-terminal sequence of RNA1 in these clones, in contrast, seemed to be the same as wild-type FHV RNA. In the clones where the 5 '-end was joined with the $3^{\prime}$-end, there was nucleotide addition. Since nucleotide addition was not observed at the $5^{\prime}$-ends examined, this suggested that nucleotide addition occurred at the 3 '-ends only. The added sequence was short and variable. This small nucleotide addition seems to be due to the terminal transferase activity of FHV replicase, which was reported to restore the nucleotide loss at the 3 '-end of the FHV RNA template [19]. We also examined the terminal sequences of the replication products in $N$. benthamiana leaves inoculated with pCB FHV(eGFP)-CP construct. In this case, as shown in Fig. 4C, 10 out of 12 clones of 5 '-end sequences had deletions and the deletion was more extensive compared to the replication products in BHK cells. This may explain the observation that FHV RNA1 replication was inefficient compared to FHV RNA3 replication in $N$. benthamiana. The $3^{\prime}$-terminal sequences were identical to the wild-type sequence, as in BHK cells.

\section{Discussion}

In this study, we demonstrated that FHV RNA1 can replicate in mammalian cells despite the presence of a long heterologous sequence at the $3^{\prime}$-end. This is an important point because FHV RNA is not encapsidated into TYMV without the TYMV CP sequence. Sequence analysis of replication products suggested that the FHV replicase can correctly select the initiation site for (-)-RNA synthesis, even though the sequence element for replication initiation resides internally. Obviously, the TYMV CP sequence did not interfere with the recognition of FHV replication 
A

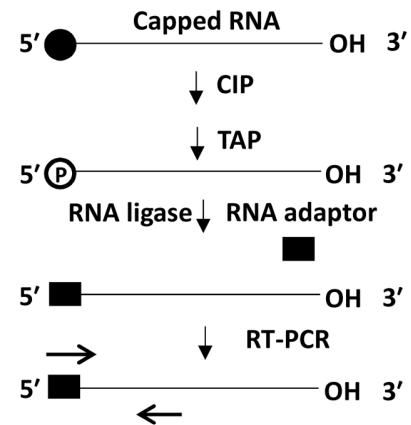

$5^{\prime}-3^{\prime}$ RLM-RACE

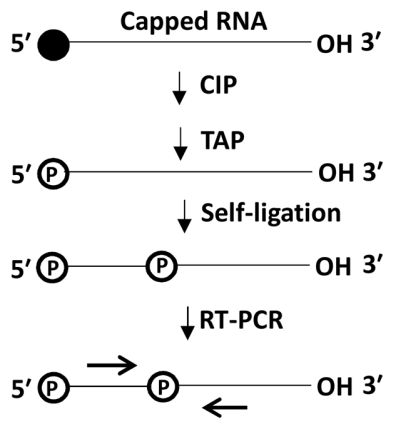

B $5^{\prime}$-ends of replicated FHV RNA1 in BHK cells $\begin{array}{llll}\text { wt } & 5 & \text { GITTGAAACAAATAAAACAGAAAAGCGAACCTAAACAATGACTCT } \cdots & 3 \\ 1-6 & \text { GTTTGAAACAAATAAAACAGAAAAGCGAACCTAAACAATGACTCT } \cdots & 3 \\ 7-9 & \text {---TGAAACAAATAAAACAGAAAAGCGAACCTAAACATGACTCT } \cdots & 3 \\ 10 & & --- \text { GAAACAAATAAAACAGAAAAGCGAACCTAAACAATGACTCT } \cdots & 3\end{array}$,

3' and 5'-ends of replicated FHV RNA1 in BHK cells 3 '-end $\quad 5^{\prime}$-end

C 5'-ends of replicated FHV RNA1 in $\mathbf{N}$. benthamiana

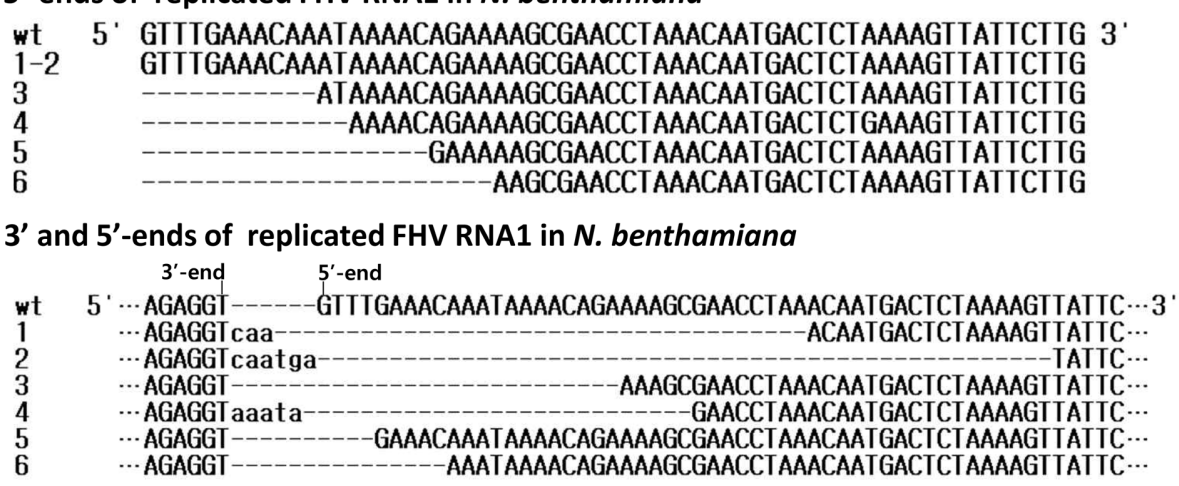

Fig. 4. Sequence analysis of replication products of FHV(eGFP)-CP.

(A) Schematic representation. Total RNA samples were treated with CIP to remove free $5^{\prime}$-phosphates from RNA molecules. The RNA sample was then treated with TAP to remove $5^{\prime}$ cap structures, leaving monophosphates at the $5^{\prime}$-ends of RNA species. To examine the $5^{\prime}$-end sequences of FHV RNA1, a synthetic RNA adaptor was ligated to the RNA population using T4 RNA ligase. For 5'-3' RLM-RACE, TAP-treated RNAs were self-ligated without RNA adaptors. cDNA was generated by reverse transcription, then amplified and examined by sequence analysis. (B) and (C) Sequence alignment of the FHV RNA1 replication products isolated from BHK cells (B) or N. benthamiana leaves (C). Sequence of unknown origin is indicated by lowercase.

signals by FHV replicase.

An in vitro assay of FHV replicase activity showed that deletion was more crucial at the $5^{\prime}$-end than the 3 '-end [19]. (-)-RNA synthesis initiation tolerated the loss of two nucleotides at the 3 '-end. RNA synthesis still occurred upon more extensive nucleotide loss (13-29 nucleotides), although synthesis was somewhat inhibited. In contrast, a two-nucleotide loss at the 5'-end significantly affected the synthesis of (+)-RNA, while loss of three or more nucleotides resulted in complete inhibition of the replicase. This indicated that the 5'-terminal sequence, but not the $3^{\prime}$ terminal sequence, is crucial to the recognition capacity of 
FHV replicase. In spite of this, the results of the sequence analysis in this study showed that FHV replicase might have difficulty in recognizing the start site at the $5^{\prime}$-end, because deletion was frequently observed at the $5^{\prime}$-ends of the RNA1 products.

FHV RNA replication was lower at $30^{\circ} \mathrm{C}$ compared with $28^{\circ} \mathrm{C}$. However, the reduction of RNA replication was far smaller compared with the large decrease in $\mathrm{B} 2$ protein levels at $30^{\circ} \mathrm{C}$ (Figs. 2B and $3 \mathrm{~B}$ ). This finding suggests that B2 protein does not play a role in FHV RNA replication, and is consistent with a previous report that FHV RNA replication in BHK cells is vigorous in the absence of B2 [5]. The decrease in FHV RNA replication at $>28^{\circ} \mathrm{C}$ was previously reported by Ball et al. [20]. Probably, the temperature dependence of RNA replication is ascribed to the enzymatic properties of FHV replicase.

The results represented in Fig. 3 show that synthesis of B2-eGFP protein in BHK21 cells was very low at $30^{\circ} \mathrm{C}$, although there was a sufficient amount of RNA. Petrillo et al. [15] showed that B2 protein protected FHV RNA2, which would become trapped in the subcellular structures where translational silencing occurs in the absence of B2. They also hypothesized that $\mathrm{B} 2$ would also protect its own mRNA, FHV RNA3, based on the observation that the introduction of mutations at the double-stranded RNA binding domain of B2 drastically reduced synthesis of not only viral coat protein but also B2. If B2 influences its own synthesis, a small change in B2 activity could eventually lead to a large difference in B2 protein levels through an amplification process. A small decrease in B2 activity would reduce the synthesis of B2. This would decrease B2 activity further, leading to even more reduced B2 synthesis. We hypothesized that the B2 protein followed this scenario at $30^{\circ} \mathrm{C}$. Another interesting observation was that some, though scant in number, BHK21 cells expressed eGFP strongly at $30^{\circ} \mathrm{C}$ (Fig. 3). In this small group of transfected cells, although at a very low probability, the B2-eGFP proteins that were synthesized more often than average by chance at early times of transfection may have tipped the balance in the direction of enhanced synthesis of B2-eGFP at this temperature.

As previously mentioned, injection of recombinant FHV RNA1 containing an eGFP gene elicited a humoral immune response in mice [9]. This suggests that the FHV RNA1 can replicate and express the transgene at $37^{\circ} \mathrm{C}$. However, the immune response was weak and was also anticipated from our observation (Fig. 3). Weak transgene expression can be overcome by employing nodamura virus $(\mathrm{NoV})$, another member of the Nodaviridae family. Similar to FHV, NoV can replicate in mammalian cells. Unlike FHV, NoV replicase is active at $37^{\circ} \mathrm{C}$ [20]. Transgene expression can also be enhanced by a modified expression system. The results reported by Petrillo et al. [15] and this study show that translation of FHV RNA2 and RNA3 is influenced by B2 protein, which is highly sensitive to temperature (Fig. 4). On the contrary, translation of FHV RNA1 is not affected by B2 [15]. Furthermore, Albarino et al. [17] previously reported that a truncated FHV RNA can replicate well as long as the RNA has the 297-nucleotide and 108-nucleotide of the 5'- and 3'-terminal sequences of FHV RNA1, respectively. Based on this observation, a bipartite expression vector system can be devised, where one RNA provides a transgene with the minimal replication signals of FHV RNA1 and the other supplies FHV (or NoV) replicase.

\section{Acknowledgments}

This study was supported by the Basic Science Research Program through the National Research Foundation of Korea (NRF) funded by the Ministry of Education, Science, and Technology (NRF-2010-0021868).

\section{Conflict of Interest}

The authors have no financial conflicts of interest to declare.

\section{References}

1. Shin HI, Kim IC, Cho TJ. 2008. Replication and encapsidation of recombinant Turnip yellow mosaic virus RNA. BMB Rep. 41: 739-744.

2. Dreher TW. 2004. Turnip yellow mosaic virus: transfer RNA mimicry, chloroplasts and a C-rich genome. Mol. Plant Pathol. 5: 367-375.

3. Kim HB, Kim DY, Cho TJ. 2014. Replication and packaging of Turnip yellow mosaic virus RNA containing Flock house virus RNA1 sequence. BMB Rep. 47: 330-335.

4. Johnson KL, Ball LA. 1999. Induction and maintenance of autonomous Flock house virus RNA1 replication. J. Virol. 73: 7933-7942.

5. Ball LA. 1995. Requirements for the self-directed replication of Flock house virus RNA1. J. Virol. 69: 720-727.

6. Li H, Li WX, Ding SW. 2002. Induction and suppression of RNA silencing by an animal virus. Science 296: 1319-1321.

7. Chao JA, Lee JH, Chapados BR, Debler EW, Schneemann A, Williamson JR. 2005. Dual Modes of RNA-silencing suppression by Flock house virus protein B2. Nat. Struct. Mol. Biol. 12: 952-957. 
8. Smith ML, Carbo T, Bernales J, Lindbo JA, Pogue GP, Palmer KE, et al. 2007. Assembly of trans-encapsidated recombinant viral vectors engineered from Tobacco mosaic virus and Semliki Forest virus and their evaluation as immunogens. Virology 358: 321-333.

9. Zhou Y, Maharaj PD, Mallajosyula JK, McCormick AA, Kearney CM. 2015. In planta production of flock house virus transencapsidated RNA and its potential use as a vaccine. Mol. Biotechnol. 57: 325-336.

10. Azizgolshani O, Garmann RF, Cadena-Nava R, Knobler CM, Gelbart WM. 2013. Reconstituted plant viral capsids can release genes to mammalian cell. Virology 441: 12-17.

11. Schirawski J, Voyatzakis A, Zaccomer B, Bernardi F, Haenni AL. 2000. Identification and functional analysis of the turnip yellow mosaic tymovirus subgenomic promoter. J. Virol. 74: 11073-11080.

12. Kim D, Cho TJ. 2017. Replication of recombinant flock house virus RNA encapsidated by turnip yellow mosaic virus coat proteins in Nicotiana benthamiana. J. Bacteriol. Virol. 47: 84-95.

13. Kim D, Lee Y, Dreher TW, Cho TJ. 2018. Empty Turnip yellow mosaic virus capsids as delivery vehicles to mammalian cells. Virus Res. 252: 13-21.
14. Cho TJ, Dreher TW. 2006. Encapsidation of genomic but not subgenomic Turnip yellow mosaic virus RNA by coat protein provided in trans. Virology 356: 126-135.

15. Petrillo JE, Venter PA, Short JR, Gopal R, Deddouche S, Lamiable O, et al. 2013. A. Schneemann, Cytoplasmic granule formation and translational inhibition of nodaviral RNAs in the absence of the double-stranded RNA binding protein B2. J. Virol. 87: 13409-13421.

16. Maharaj PD, Mallajosyala JK, Lee G, Phillip T, Zhou Y, Kearney CM, et al. 2014. Nanoparticle encapsidation of flock house virus by auto assembly of tobacco mosaic virus coat protein. Int. J. Mol. Sci. 15: 18540-18556.

17. Albarino CG, Eckerle LD, Ball LA. 2003. The cis-acting replication signal at the 3' end of Flock house virus RNA2 is RNA3-dependent. Virology 311: 181-191.

18. Eckerle LD, Albarino CG, Ball LA. 2003. Flock house virus subgenomic RNA3 is replicated and its replication correlates with transactivation of RNA2. Virology 317: 95-108.

19. Wu W, Wang Z, Xia H, Liu Y, Qiu Y, Liu Y, et al. 2014. Flock house virus RNA polymerase initiates RNA synthesis de novo and possesses a terminal nucleotidyl transferase activity. PLoS One 9: e86876.

20. Ball LA, Amann JM, Garrett BK. 1992. Replication of Nodamura virus after transfection of viral RNA into mammalian cells in culture. J. Virol. 66: 2326-2334. 